\title{
Numerical Approximation of Plane Deformation for the Indentation of Granular Material by a Smooth Rigid Wedge Punch
}

\author{
Syafikah Ayob*, Nor Alisa Mohd Damanhuri \\ Faculty of Industrial Sciences \& Technology, University Malaysia Pahang, Malaysia
}

Copyright $(2019$ by authors, all rights reserved. Authors agree that this article remains permanently open access under the terms of the Creative Commons Attribution License 4.0 International License

\begin{abstract}
In this paper, a numerical approximation of the stress equation for the indentation of granular materials by a smooth rigid wedge is presented. Plane strain conditions are assumed, and the materials obey the Mohr-Coulomb yield condition. This method determines the deformation of granular material under a smooth rigid wedge punch and construction of stress field in the deforming region which are presented by using MATLAB programme. The granular material is assumed to be in dense, solid like state. The solution only refers to the initial motion after the punch. This was then applied on one type of boundary value problem. By using MATLAB, the value of each point $(x, y)$ and the stress variables $(p, \psi)$ which construct the deformation field are calculated. This method provides simple and reliable algorithms for the solution of the deformation problems involving the stress variables. The results will consequently help in the improvement of the existing labs and experimental facilities in the industries and will eventually increase its efficiency.
\end{abstract}

Keywords Deformation, Granular Material, Wedge Indentation

\section{Introduction}

The formulation of equations which govern the deformation and the flow of granular are outstanding problems in continuum mechanics. A review of a foundation theory in plasticity was made by [8]. This foundation is made based on the assumption of coaxiality of the principal stress and rate tensor. He proposed the boundary value problem for the numerical solution. By abandoning the assumption of coaxiality, an alternative family of models has been derived based on a kinematic hypothesis involving the concepts of shearing motion parallel to a surface, rotation of that surface, and dilation or contraction normal to the surface. One such model is the double-shearing theory, originally proposed by [7] for incompressible flows. The theory has been applied to the problem for the indentation of semi-infinite mass of soils by a smooth rigid flat punch. In this theory, the characteristic curves for the stresses and velocities coincide, and every deformation is assumed to consist of simultaneous shears along the two families of stress characteristics.

By applying the double slip and double spin model that was given by [14], the numerical approximation to the solution for double-slip and double-spin model for the deformation and flow of granular materials has been solved by [5]. The numerical approximation obtained then applied to some planar problems. The problem is considered is the indentation of granular material by a smooth rigid flat punch. Therefore, by applying Hill's boundary value problem and the slip line, theory and solution by [5], [7], [4] and [14], the aim of this study is to develop a numerical method to find the approximations for the indentation by a smooth rigid wedge punch for the granular materials. 


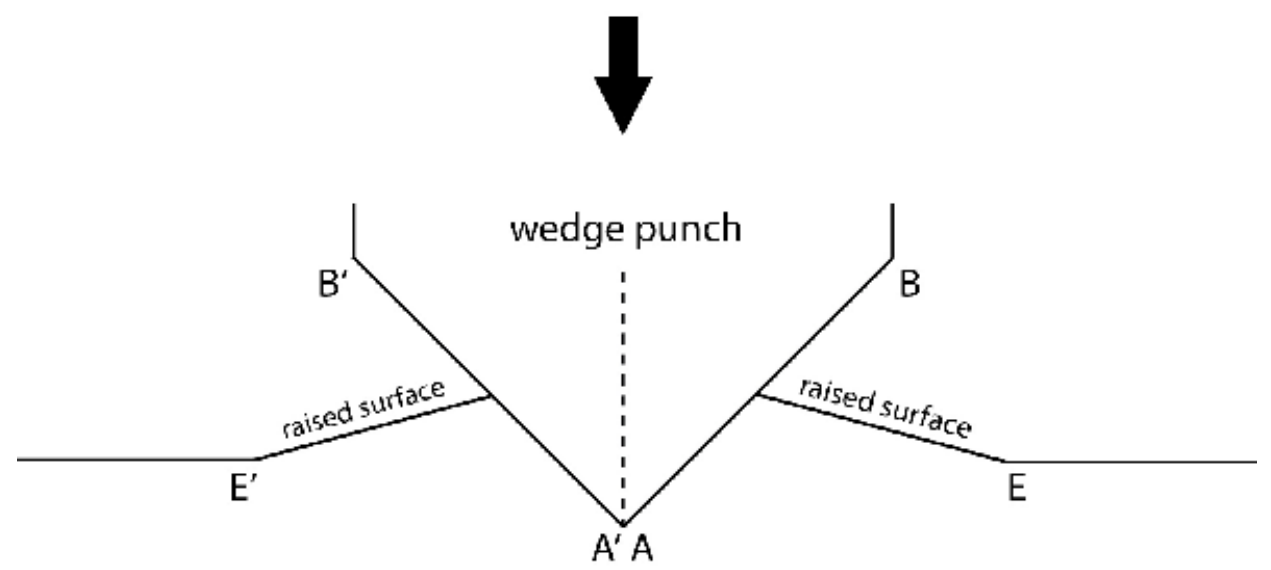

Figure 1. Indentation by smooth rigid wedge

The problem considered here is the indentation of a granular material by a smooth rigid wedge punch under the condition of plane strain. The behaviour of a granular material under an indenting rigid body is a fundamental problem with wide applications especially in engineering activities. This method provides simple and reliable algorithms for the solution of the granular deformation problem.

Shield[1] proposed a solution for the normal indentation of a granular material by a smooth rigid wedge in which the analysis is based on the assumption that the granular material obeys the Mohr-Coulomb yield criterion and its associated flow rule. The extension of indentation of a granular material by a smooth rigid wedge was presented in [2]\{ TA \ "Tordesillas \& Shi (1999)" \s "Tordesillas \& Shi (1999)" \c 1 \}. The authors presented a solution to the normal plane strain indentation of a double shearing dilatant granular material by a smooth rigid wedge. In this paper, we construct a numerical method of constructing stress deformation fields by using MATLAB program. In section 2, we summarize the basic governing equations for the plane deformations, while in the next section, we discuss the numerical method for the basic construction of stress characteristics field by using MATLAB program.

\section{The Governing Equation for the Plane Deformation}

In this section, the governing equations for the plane deformation of granular materials are presented. The equilibrium equations are given by

$$
\begin{array}{r}
\frac{\partial \sigma_{11}}{\partial x}+\frac{\partial \sigma_{12}}{\partial y}=0 \\
\frac{\partial \sigma_{12}}{\partial x}+\frac{\partial \sigma_{22}}{\partial y}=0
\end{array}
$$

with Mohr Coulomb yield criterion

$$
\tau=c-\sigma_{n} \tan \phi
$$

where $c$ is the cohesion, $\sigma_{n}$ is the stress component and $\phi$ is the internal friction of the material. Relative to the $\alpha$ an $\beta$-characteristic lines, the stress characteristic directions are given by

$$
\begin{aligned}
& \frac{d y}{d x}=\tan \left(\psi-\frac{\pi}{4}-\frac{\phi}{2}\right) \\
& \frac{d y}{d x}=\tan \left(\psi+\frac{\pi}{4}+\frac{\phi}{2}\right)
\end{aligned}
$$

which leads to the following governing stress equations for $p$ and $\psi$ given by

$\cos \phi \frac{\partial p}{\partial s_{\alpha}}+2 q \frac{\partial \psi}{\partial s_{\alpha}}=0$ along the $\alpha$-characteristic

and

$\cos \phi \frac{\partial p}{\partial s_{\beta}}-2 q \frac{\partial \psi}{\partial s_{\beta}}=0$ along the $\beta$-characteristic

where $\psi$ represents the angle between the principal stress and the $x$-axis

$$
\begin{gathered}
p=-\frac{1}{2}\left(\sigma_{11}+\sigma_{22}\right) \\
q=\frac{1}{2}\left(\sigma_{11}-\sigma_{22}\right)
\end{gathered}
$$

Also, $\frac{\partial}{\partial s_{\alpha}}, \frac{\partial}{\partial s_{\beta}}$ are directional derivatives along the $\alpha$ and $\beta$-characteristic lines. 


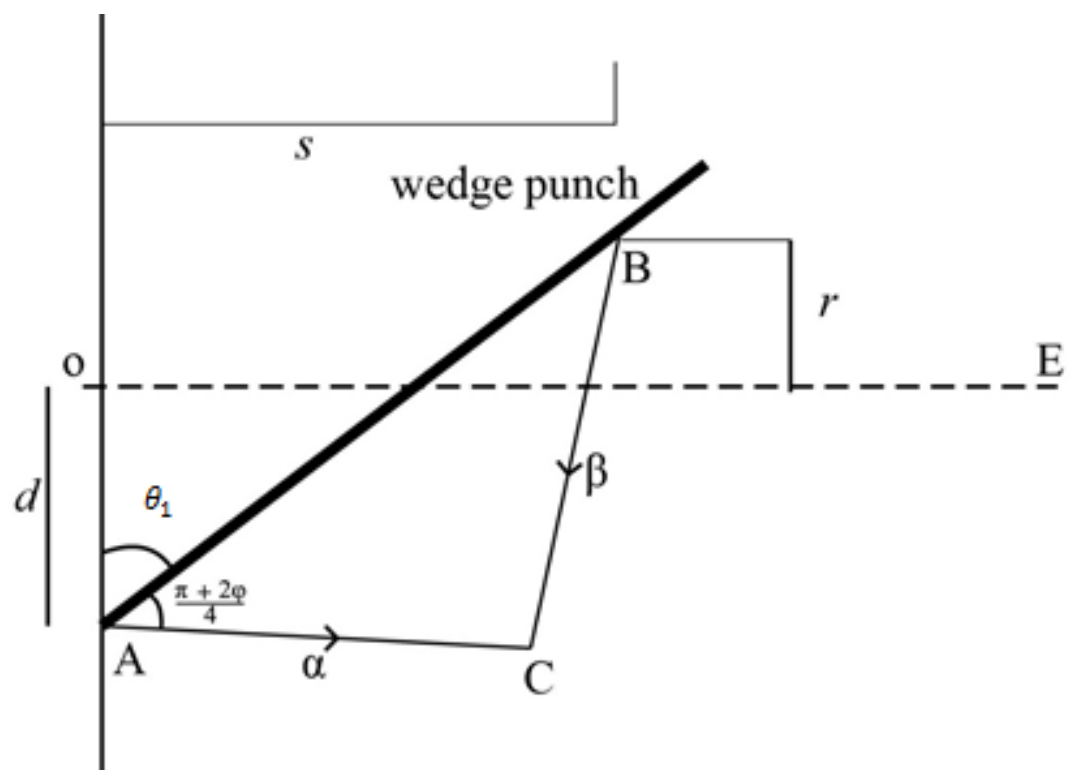

Figure 2. Deformation region under the punch

\section{Numerical Construction of the Stress} Field

The stress field in the deformation region $\mathrm{ABC}$ under the contact surface is constructed by the network of intersection between $\alpha$-characteristic line $\mathrm{AC}$ and $\beta$-characteristic line $\mathrm{BC}$ as shown in Figure 2. Suppose that the smooth rigid wedge with semi-angle $\theta_{1}=45^{\circ}$ is indented into the granular material surface $\mathrm{OE}$. The $\alpha$-characteristic line $\mathrm{AC}$ and $\beta$-characteristic line $\mathrm{BC}$ meets the contact surface at $\frac{\pi+2 \phi}{4}$. The surface at $x>s$ is a stress-free surface which implies that the surface granular material is not subject to any force. The characteristics of granular material used in this study have the angle of internal friction, $\phi=\frac{\pi}{6}$ and the cohesion, $c=2$. The depth of the penetration $\mathrm{OA}$ is denoted by $d$, while the distance between the free surface $\mathrm{OE}$ and $\mathrm{B}$ is denoted by $r$. The pressure distributions on the contact surface $\mathrm{AB}$ are assumed to be known. The surface $\mathrm{AB}$ is divided into $(n-1)$ equal parts and each point are labelled as $\operatorname{ABC}(i, 1)$ where $i=1,2,3, \ldots, n$. The coordinates $(x, y)$ and the stress variables $(p, \psi)$ are given along the contact surface $\mathrm{AB}$. In this surface, following from [3], we shall assume that the stresses are constant, therefore the solution for $p$ and $\psi$ will yield a constant value. Then, from the known coordinates and the stress variables on $\mathrm{AB}$, the stress field in the whole region $\mathrm{ABC}$ can be constructed numerically.

\section{The Numerical Approximation of the Stress Field}

The point $\mathrm{ABC}(1,1)$ and $\mathrm{ABC}(2,1)$ are the neighboring points on the contact surface $A B$, and the intersection of a $\beta$-characteristic line that passes through $\operatorname{ABC}(1,1)$ and the $\alpha$-characteristic line passing through $\mathrm{ABC}(2,1)$ is labelled as $\mathrm{ABC}(1,2)$ as shown in Figure 3. 


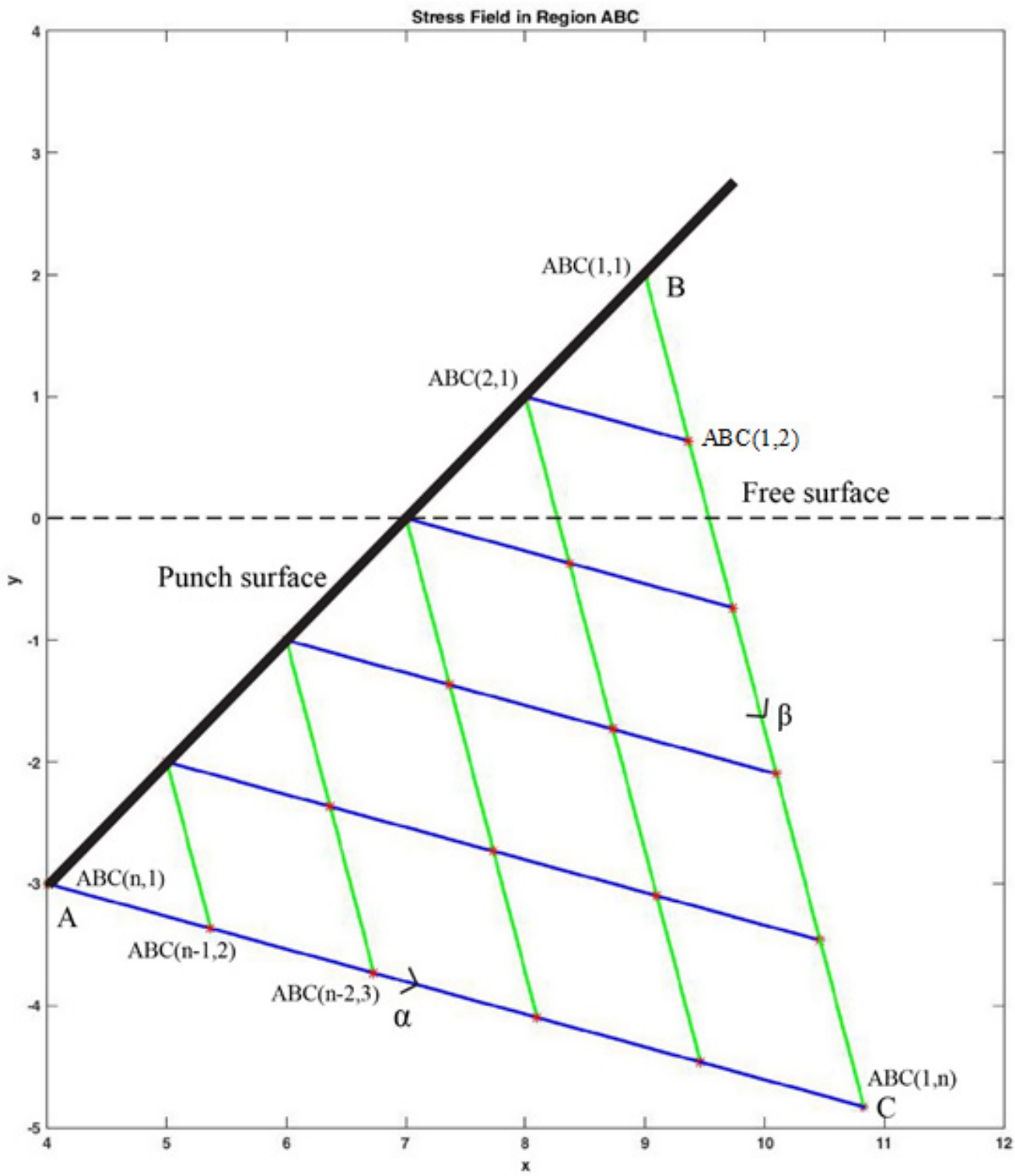

Figure 3. Matlab Construction of Stress deformation region by smooth rigid wedge.

The coordinates and the stress variables, $p$ and $\psi$, of point $\mathrm{ABC}(1,2)$ could be defined by employing the following numerical procedure.

Let the zeroth approximation for $\psi_{A B C(1,2)}$ is taken as

$$
\psi_{A B C(1,2)}^{0}=1 / 2\left(\psi_{A B C(2,1)}+\psi_{A B C(1,1)}\right)
$$

and approximation to $p_{A B C(1,2)}$ taken as

$$
p_{A B C(1,2)}^{0}=1 / 2\left(p_{A B C(2,1)}+p_{A B C(1,1)}\right)
$$

Let

$$
\psi_{\alpha}^{1}=1 / 2\left(\psi_{A B C(2,1)}+\psi_{A B C(1,2)}^{0}\right) \text { along the } \alpha \text { - characteristic line } A B C(2,1) A B C(1,2)
$$

and

$$
\psi_{\beta}^{1}=1 / 2\left(\psi_{\mathrm{ABC}(1,1)}+\psi_{\mathrm{ABC}(1,2)}^{0}\right) \text { along the } \beta \text { - characteristic line } A B C(1,1) A B C(1,2) .
$$

Then, the coordinates at point $\mathrm{ABC}(1,2)$ are given by 


$$
\begin{gathered}
x_{A B C(1,2)}^{1}=\frac{x_{A B C(1,1)} \tan \left(\psi_{\beta}^{1}+\left(\frac{\pi+2 \phi}{4}\right)\right)-x_{A B C(2,1)} \tan \left(\psi_{\alpha}^{1}-\left(\frac{\pi+2 \phi}{4}\right)\right)+y_{A B C(2,1)}-y_{A B C(1,1)}}{\tan \left(\psi_{\beta}^{1}+\left(\frac{\pi+2 \phi}{4}\right)\right)-\tan \left(\psi_{\alpha}^{1}-\left(\frac{\pi+2 \phi}{4}\right)\right)} \\
y_{A B C(1,2)}^{1}=\frac{\left(x_{A B C(1,1)}-x_{A B C(2,1)}\right) \tan \left(\psi_{\beta}^{1}+\left(\frac{\pi+2 \phi}{4}\right)\right) \tan \left(\psi_{\alpha}^{1}-\left(\frac{\pi+2 \phi}{4}\right)\right)+y_{A B C(2,1)} \tan \left(\psi_{\beta}^{1}+\left(\frac{\pi+2 \phi}{4}\right)\right)-y_{A B C(1,1)} \tan \left(\psi_{\alpha}^{1}-\left(\frac{\pi+2 \phi}{4}\right)\right)}{\tan \left(\psi_{\beta}^{1}+\left(\frac{\pi+2 \phi}{4}\right)\right)-\tan \left(\psi_{\alpha}^{1}-\left(\frac{\pi+2 \phi}{4}\right)\right)}
\end{gathered}
$$

and the stress variables $\left(\psi_{A B C(1,2)}, p_{A B C(1,2)}\right)$ are given by

$$
\begin{gathered}
\psi_{A B C(1,2)}^{1}=\frac{\left(p_{A B C(2,1)}-p_{A B C(1,1)}\right) \cos \phi+2 q_{\alpha}^{1} \psi_{A B C(2,1)}+2 q_{\beta}^{1} \psi_{A B C(1,1)}}{2 q_{\alpha}^{1}+2 q_{\beta}^{1}} \\
p_{A B C(1,2)}^{1}=\frac{p_{A B C(2,1)} \cos \phi+2 q_{\alpha}^{1}\left(\psi_{A B C(2,1)}-\psi_{A B C(1,2)}^{1}\right)}{\cos \phi}
\end{gathered}
$$

where

$$
\mathrm{q}_{\alpha}^{1}=1 / 2\left(\mathrm{p}_{\mathrm{ABC}(2,1)}+\mathrm{p}_{\mathrm{ABC}(1,2)}^{0}\right) \sin \phi+\mathrm{c} \cos \phi \text { along the } \alpha \text { - characteristic line } A B C(2,1) A B C(1,2)
$$

and

$$
\mathrm{q}_{\beta}^{1}=1 / 2\left(\mathrm{p}_{\mathrm{ABC}(1,1)}+\mathrm{p}_{\mathrm{ABC}(1,2)}^{0}\right) \sin \phi+\mathrm{c} \cos \phi \text { along the } \beta \text { - characteristic line } A B C(1,1) A B C(1,2)
$$

In order to find the solution for the coordinates $(x, y)$ and the stress variables $(p, \psi)$ at point $\mathrm{ABC}(1,2)$, an iterative procedure is required. After the $(i+1)^{t h}$ iteration, the approximation for $\left(x_{A B C(1,2)}, y_{A B C(1,2)}, \psi_{A B C(1,2)}, p_{A B C(1,2)}\right)$ is obtained. Once the point $\mathrm{ABC}(1,2)$ is constructed, then by considering the next two neighbouring points on the contact surface $\operatorname{ABC}(2,1)$ and $\operatorname{ABC}(3,1)$, the point $\operatorname{ABC}(2,2)$ is defined. This procedure is repeated until the intersection point $\operatorname{ABC}(1, n)$ is obtained. Thus, from the contact surface $\mathrm{AB}$, we construct the stress field $\mathrm{ABC}$ by using Matlab as shown in Figure 3.

\section{Discussion and Conclusions}

The solution for stress fields depend on the angle determined by the wedge semi-angle, $\theta_{1}$. When the wedge is penetrated into the granular material surface, the particles move downwards. Since the values of $\psi$ and $p$ along the punch surface are constant, then the triangular region under the punch surface $\mathrm{ABC}$, as in Figure 3, is formed where the stresses are all constants. The computed values of $\psi$ and $p$ in region $\mathrm{ABC}$ are given as follows

$$
\psi=0.7854, p=13.016
$$

These results obtained were compared to the analytical solutions given in [4] and it obeys the solution. The granular material is assumed to be in dense, solid like state. The solution only refers to the initial motion after the punch and the numerical method for stress field under the punch surface described in this paper is simple and reliable. This was then applied to one type of boundary value problem and can be constructed to other types of boundary value problems in plastic flow. This will be a part of our future work.

\section{Acknowledgements}

We would like to thank the University Malaysia Pahang for funding this research under UMP internal grant RDU170355.

\section{REFERENCES}

[1] Shield, R. T. (1953). Mixed boundary value problems in soil mechanics. Quarterly of Applied Mathematics, 11(1), 6175.

[2] Tordesillas, A., \& Shi, J. (1999, January). Frictional indentation of dilatant granular materials. In Proceedings of the Royal Society of London A: Mathematical, Physical and Engineering Sciences (Vol. 455, No. 1981, pp. 261-283). The Royal Society.

[3] Hill, R., Lee, E. H., \& Tupper, S. J. (1947). The Theory of Wedge Indentation of Ductile Materials. Proceedings of the Royal Society A: Mathematical, Physical and Engineering Sciences, 188(1013), 273-289

[4] A. Tordesillas and J. Shi, "Indentation of a double shearing dilatant granular material by a smooth rigid wedge," in Quarterly Journal of Mechanics and Applied Mathematics, vol. 51, no. 4, pp. 633-646, Nov. 1998.doi: 10.1093/qjmam/51.4.633

[5] Mohd. Damanhuri, N. A. The Numerical Approximation to Solutions for The Double-Slip and Double-Spin Model for The Deformation and Flow of Granular Materials, Ph. D. thesis, The University of Manchester, United Kingdom, 2015.

[6] Anand, L. (1983). Plane deformations of ideal granular materials Journal of the Mechanics and Physics of Solids, 31(2), 105-122.

[7] Spencer, A. J. M. (1964). A theory of the kinematics of ideal 
soils under plane strain conditions. Journal of the Mechanics and Physics of Solids, 12(5), 337-351.

[8] R. Hill. (1950). The Mathematical Theory of Plasticity, 1.

[9] Harris D. (2006) Double-Slip and Spin: Dilatant Shear in a Reduced Cosserat Model. In: Wu W., Yu HS. (eds) Modern Trends in Geomechanics. Springer Proceedings in Physics, vol 106. Springer, Berlin, Heidelberg.

[10] Spencer, A. J. M. (1982). Deformation of ideal granular materials. In Mechanics of solids: the Rodney Hill 60th anniversary volume (pp. 607-652).

[11] Harris, D. (1985). On the numerical integration of the stress equilibrium equations governing the ideal plastic plane deformation of a granular material. Acta mechanica, 55(3-4), 219-238.

[12] Harris, D. Acta Mechanica (2001) 146: 199. https://doi.org/10.1007/BF01246733.

[13] Damanhuri, N. A., \& Ayob, S. (2017). A general numerical approximation of construction of axisymmetric ideal plastic plane deformation of a granular material J. Phys.: Conf. Ser. 890012059

[14] Harris D., Grekova E.F.: A hyperbolic well-posed model for the flow of granular materials. J. Eng. Math. 52, 107135 (2005).

[15] Harris, D. Acta Mech (2014) 225: 2277. https://doi.org/10.1007/s00707-014-1129-x. 